\title{
German an Lichtfreund. Die Hyperboreischen Briefe und das preußische Religionsedikt (9.7.1788)
}

German an Lichtfreund. Le périodique Hyperboreische Briefe et l'édit de censure religieuse en Prusse (9 juillet 1788)

German to Lichtfreund. The Hyperboreischen Briefe and the Prussian

Religious Edict (9. 7. 1788)

\section{Christina Stange-Fayos}

\section{(2) OpenEdition}

\section{Journals}

Édition électronique

URL : http://journals.openedition.org/ceg/887

DOI : $10.4000 /$ ceg. 887

ISSN : 2605-8359

\section{Éditeur}

Presses Universitaires de Provence

Édition imprimée

Date de publication : 30 mai 2016

Pagination : 83-98

ISBN : 979-10-320-0067-0

ISSN : 0751-4239

\section{Référence électronique}

Christina Stange-Fayos, «German an Lichtfreund. Die Hyperboreischen Briefe und das preußische Religionsedikt (9.7.1788) », Cahiers d'Études Germaniques [Online], 70 | 2016, Online erschienen am: 17 Dezember 2017, abgerufen am 26 November 2020. URL : http://journals.openedition.org/ceg/887 DOI : https://doi.org/10.4000/ceg.887 


\section{German an Lichtfreund \\ Die Hyperboreischen Briefe und das preußische Religionsedikt (9.7.1788)}

Christina STANGE-FAYOS

Université Toulouse Jean-Jaurès

Die Gegenden nach Westen und Nordwesten, jenseits des Adriatischen Meeres, nannten die Griechen die Hyperboreischen, d.h. über dem Winde belegenen, versteht sich von Griechenland und Kleinasien aus. Danach nannte man auch später die Völker: Hyperboräer. ${ }^{1}$

Brief und Presse waren im 18. Jahrhundert eng verbunden. Die Zeitungen wurden von Druckern hergestellt, die darin die Möglichkeit sahen, ihre Druckerpressen fortlaufend zu nutzen. Den Stoff - die Nachrichten - bezogen sie über die Post von Korrespondenten, die aus verschiedenen Ländern Europas Briefe über wichtige Begebenheiten schrieben. Wenn damals jemand journalistisch arbeitete, dann jene Briefschreiber, die Nachrichten vor Ort sammelten und sie gewerbsmäßig weitergaben. Dieter Paul Baumert hat diese Phase den „korrespondierenden Journalismus“ genannt. ${ }^{2}$ Der korrespondierende Journalist bemühte sich in der Regel um nüchterne Informationen und Faktenschilderung. Diese Art von Journalismus begründete das Ansehen der Zeitung und machte sie zum erfolgreichsten Presseorgan Europas im späten 18. Jahrhundert. Wurde das Bild des unparteiischen Publizisten hervorgehoben, dann gerade weil es trotzdem immer wieder zu Meinungsäußerungen in den Zeitungen kam. Die Nachrichten und Berichte waren keineswegs frei von Bewertungen. Obwohl

1. Georg Friedrich König, Deutsche Briefe: geschrieben im Zuchthaus zu Emden, Emden, Rakebrand, 1837, Erstes Heft, S. 28.

2. „Der korrespondierende Journalismus, der sich im nicht-öffentlichen Briefwechsel vorgeformt hatte, prägte dann den Inhalt der Zeitung, seitdem diese zu Beginn des 17. Jahrhunderts voll ausgebildet war. Dabei verstanden sich die Korrespondenten als reine Berichterstatter. Es kam ihnen darauf an, zu informieren, Geschehnisse und Sachverhalte möglichst getreu wiederzugeben. Dies geschah keineswegs nur mit Rücksicht auf die amtliche Zensur, der die Zeitungen - wie alle Druckwerke - unterworfen waren. Der Verzicht auf Meinungsäußerung entsprach vielmehr dem journalistischen Ideal und Selbstverständnis der Zeit.“, Dieter Paul Baumert, Die Entstehung des deutschen Journalismus. Eine sozialgeschichtliche Studie, München und Leipzig, Duncker, 1928, S. 18 und 29 ff. Hervorhebung C. S.-F. 
der korrespondierende Journalismus im 18. Jahrhundert vorherrschend blieb, trat noch in jener Zeit, aus geistes- und sozialgeschichtlichen Gründen, ein neuer Typ von Journalist auf. Die Aufklärung war am Werk und mit ihr die Forderung nach Pressefreiheit. Der Beruf des freien Schriftstellers bildete sich heraus (Lösung aus der fürstlichen Patronage); in zunehmender Zahl machten Autoren den Versuch, aus dem Einkommen schriftstellerischer Arbeit zu leben. ${ }^{3}$ Zeitungen und Zeitschriften boten diesbezüglich den Vorteil, durch ihr periodisches Erscheinen laufend Einkünfte abzuwerfen. So betätigten sich viele Literaten dieser Zeit zugleich als Journalisten. Aufgrund dieser sozialgeschichtlichen Veränderungen hat man die den korrespondierenden Journalismus ablösende Phase mit dem Etikett „schriftstellerischer Journalismus“ versehen. ${ }^{4}$ Nach dieser „Wende“ konnte das Briefliche in der Presse jedoch weiterhin eine wichtige - wenn auch unterschiedliche - Rolle spielen, wie im Laufe dieses Beitrags über die Hyperboreischen Briefe gezeigt werden wird.

Die Hyperboreischen Briefe wurden zwischen 1788 und 1790 von Wilhelm Ludwig Wekhrlin (1739-1792) herausgegeben, der beispielhaft für den schriftstellerischen Journalismus steht. Zwölf Jahre lang hat er fast ausschließlich von seinen Publikationen gelebt, was zu dieser Zeit äußerst selten war. ${ }^{5}$ Als Sohn eines Landpfarrers, aus einer alten württembergischen Familie, besuchte er das Gymnasium in Stuttgart. ${ }^{6}$ Gern behauptete er, seine berufliche Laufbahn als Privatsekretär Voltaires in Frankreich begonnen zu haben; eigentlich arbeitete er als Schreiber in Ludwigsburg. Allerdings war er sehr frankophil, sein geistiges Vorbild war tatsächlich Voltaire, dessen Werke er auszugsweise ins Deutsche übersetzte und publizierte. Von 1766-1776 lebte er in Wien und versuchte sich dort an handgeschriebenen, geheimen Zeitungen, was ihm Probleme mit der österreichischen Zensur bereitete. Er wurde aus dem Habsburgerreich ausgewiesen. Nach kurzem Aufenthalt in Regensburg kehrte er jedoch nach Wien zurück und trat in den Dienst der französischen Botschaft. Eine Spionageaffäre wurde ihm zum Verhängnis: endgültig ausgewiesen, übersiedelte er 1776 nach Augsburg, ab 1777 nach Nördlingen, wo seine erste satirische Reisebeschreibung Denkwürdigkeiten von Wien erschien. Gleich darauf publizierte er seine zweite Schrift Anselmus Rabiosus Reise durch Oberdeutschland (1777 bzw. 1778), die aufgrund ihres ironischen Tons nicht geringes Aufsehen erregte. ${ }^{7}$ Der Nördlinger Verleger Carl Gottlob Beck, der das Buch Ende 1777

3. Hans Jürgen Haferkorn, „Zur Entstehung der bürgerlich-literarischen Intelligenz und des Schriftstellers in Deutschland zwischen 1750 und 1800“, in Bernd Lutz (Hrsg.), Deutsches Bürgertum und literarische Intelligenz, Stuttgart, Metzler, 1974, S. 113-275.

4. Baumert, Die Entstehung des deutschen Journalismus, S. 35.

5. Jean Mondot, Wilhelm Ludwig Wekhrlin, publiciste des Lumières, Lille, Atelier national de reproduction des thèses, 1986, S. 15.

6. Friedrich Wilhelm Ebeling, „Wilhelm Ludwig Wekhrlin: Leben und Auswahl seiner Schriften. Zur Culturgeschichte des 18. Jahrhunderts“, Berlin, Köppen, 1869; http://www.literaturportalbayern.de/autorenlexikon [Stand: 12 Januar 2015].

7. "Si l'ironie mordante est son registre préféré, peut-être aussi son meilleur, il sait manier d'autre part l'invective, l'indignation, l'émotion. Il est certain en tout cas qu'il 'invente' ou qu'il 'importe' 
herausgebracht hatte, ermutigte zu weiterer Zusammenarbeit und ließ den Plan zu einer Zeitung entstehen: Wekhrlin sollte als Redakteur fungieren, Becker Drucker und Verleger sein. Wekhrlins erste Zeitung erschien ab Januar 1778 zweimal wöchentlich mit dem Titel Das Felleisen, also nach der ledernen Reisetasche der Postboten benannt - eine Brücke zum korrespondierenden Journalismus. Tatsächlich stützt er sich z.T. noch auf Briefe, wertete jedoch vor allem andere Zeitungen aus. ${ }^{8}$ Der Redakteur des Felleisens setzte sich Aufklärung zum Ziel, wofür schon das Motto „Inter Nubila Phoebus“ sprach, welches das Mittelfeld des grafischen Titelsignets (ein Raubvogel mit einem Kranz im Schnabel) beherrschte. Im Felleisen ließ sich Wekhrlin von einem neuen journalistischen Selbstverständnis leiten und bezeichnete sich selbst als „Spion des Publikums“, der es davor bewahrt, getäuscht zu werden und der ihm hilft, die geschilderten Ereignisse zu deuten.

Sein neues publizistisches Rollenverständnis musste Wekhrlin in Konflikte bringen. Zum einen wollte er sich nicht mehr einem Verleger unterordnen. Zum anderen missfiel diese Art Journalismus der städtischen Obrigkeit, die offene Stellungnahmen in den Zeitungen nicht tolerierte. Wekhrlin musste die Redaktion des Felleisens aufgeben und wurde aus der Stadt verjagt. Doch bedeutete dies nicht das Ende seines Beitrags zur Geschichte des deutschen Journalismus. Er ging in das benachbarte Baldingen und verlegte sich von der Zeitungs- auf die Zeitschriftenpresse. Diese Gattung erlaubte eher, sich ganz auf räsonierende Beiträge zu konzentrieren. ${ }^{9}$ Seine erste Zeitschrift, Die Chronologen (1779-1783, monatlich) ließ er in Nürnberg verlegen. Diese, wie die nachfolgende zweite Zeitschrift, Das graue Ungeheuer (1784-1787, auch monatlich), bei der u.a. Georg Christoph Lichtenberg und Gottfried August Bürger mitarbeiteten, waren sehr erfolgreich. Aus Wut über seine Ausweisung verfasste Wekhrlin jedoch zugleich Spottschriften gegen den Nördlinger Bürgermeister, was ihm allerdings nur den Zorn des Stadtrates und eine Festnahme durch den Fürsten von Oettingen-Wallenstein einbrachte. Am 4. Mai 1787 wurde Wekhrlin verhaftet und auf Burg Hochhaus gebracht, wo er bis 1792 ohne rechtlichen Entscheid unter Arrest blieb. Trotzdem gelang es ihm, seine Tätigkeit als Publizist fortzuführen: Von 1788-1790 erschien die Fortsetzung des Ungeheuers unter dem Titel Hyperboreische Briefe (wiederum monatlich). ${ }^{10}$ Gedruckt und verlegt wurden die drei Organe von dem Nürnberger Verleger Felßecker. Mit seiner dritten Zeitschrift Hyperboreische Briefe wechselte Wekhrlin 1788 die

en Allemagne un style dont un Heine se servira par la suite à la perfection. " (Mondot, Wilhelm Ludwig Wekhrlin, S. 268).

8. Jürgen Wilke, „Spion des Publikums, Sittenrichter und Advokat der Menschheit. Wilhelm Ludwig Wekhrlin (1739-1792) und die Entwicklung des Journalismus in Deutschland“, in Publizistik: Vierteljahreshefte für Kommunikationsforschung, Wiesbaden, Bd. 38 (1993), S. 326.

9. Ibid., S. 328.

10. Der erste Biograf Wekhrlins, sein Mitarbeiter Karl Knoblauch von Hatzbach, beschreibt die Haftbedingungen des Publizisten als äußerst angenehm: Wekhrlin sei eher Gast als Gefangener auf dem „schön und romantisch gelegenen Hochhaus“ gewesen, „dessen frische Naturumgebung er in HB II, 125,128 preist“. Karl Damian Achaz von Knoblauch zu Hatzbach, „Wekhrlin, Wilhelm Ludwig“, in Allgemeine Deutsche Biographie, Bd. 41 (1896), S. 645-653. 
Darstellungsform. Er wählte jetzt die Einkleidung eines Briefwechsels, wobei er zahlreich fiktiv fantastische Personen auftreten ließ. ${ }^{11}$ Der Herausgeber der Zeitschrift stellt sich als Sammler der Briefe dar. ${ }^{12}$ Auch inhaltlich markieren die Hyperboreischen Briefe einen Paradigmenwechsel: den Begriff „öffentliche Meinung“ benutzte Wekhrlin hier zum ersten Mal. ${ }^{13}$ Er war der Meinung, dass nichts schlimmer sein konnte, als der Missbilligung des Publikums ausgesetzt zu sein. Die Gesetzgebung stand vor dem öffentlichen Gerichtshof der Vernunft - dieser konnte seine Autorität auch gegen den Thron wenden. ${ }^{14}$ Um die Herrscher zu kontrollieren und die Leserschaft $\mathrm{zu}$ beschützen, benötigte der Journalist als Advokat der Menschheit, als „Priester der Publizität“ wie Wekhrlin sich in den Hyperboreischen Briefen darstellte ${ }^{15}$, absolute Pressefreiheit. Nun stellte der Kampf mit der Zensur damals ein gesamteuropäisches Problem dar. ${ }^{16}$ Der Thronwechsel im bislang aufgeklärten Preußen versprach in dieser Hinsicht nichts Gutes. Der kunstsinnige Prinz Friedrich Wilhelm war Mitglied des Rosenkreuzerordens und lieferte sich der Beeinflussung durch seine späteren Minister Bischoffwerder und Wöllner aus. Als die preußische Regierung 1788 wie befürchtet die Zensur mit dem Religionsedikt verschärfte und der Aufklärung den Kampf ansagte ${ }^{17}$, reagierten die Hyperboreischen Briefe sofort.

Den ersten Brief über das Religionsedikt schreibt „German an Lichtfreund“, also: Der Deutsche an den Aufklärer. ${ }^{18}$ Der Brief ist betitelt „Böse Aspekten“ und steht unter dem Zeichen des Thronwechsels in Preußen, welches daher vom Obskurantismus bedroht zu sein schien: „Nicht immer sind unsere Ahndungen Lügner. Letztlich (HB I, S. 303) meinte ich, wir dürfen mit unserer Toleranz nicht zu laut schreien: wir wären noch nicht überm Bache.“ ${ }^{19}$ Der Stil mutet

11. "La présentation des articles diffère assez sensiblement des précédentes : à peu près tous les articles sont en effet présentés sous forme épistolaire, adressées à des correspondants fictifs. Ces correspondants portent des noms évoquant tantôt l'antiquité gréco-latine, tantôt la comédie italienne ou française (ex. : Xanthus, Hippias, Clio, Melpomène, Evander, Polemon, Erast, Argant, Diogène, Kallias, Alceste, Blondin, Arabella, Arthur, Kleanth, etc.). La présentation plus soignée de la revue, agrémentée de nombreuses vignettes, contribue à faire naître une atmosphère wielandienne et rococo non dépourvue d'élégance. On le sait, cela n'empêchera pas l'échec de la revue. " (Mondot, Wilhelm Ludwig Wekhrlin, S. 269).

12. Wilke, „Spion des Publikums“, S. 330.

13. Mondot, Wilhelm Ludwig Wekhrlin, S. 246.

14. Dirk Kemper, Missbrauchte Aufklärung? Schriften zum preußischen Religionsedikt vom 9. Juli 1788, 118 Schriften auf 202 Mikrofiches, Hildesheim, Olms, 1996, Begleitband S. 70. (Der Ausdruck „Gerichtshof der öffentlichen Meinung“ stammt von Klaus Epstein, s. Klaus Epstein, Die Ursprünge des Konservativismus in Deutschland, Frankfurt a.M., Ullstein, 1973).

15. $H B$ I, 1788, S. 23, zitiert nach Wilke, „Spion des Publikums“, S. 330.

16. „Ein die Aufklärung existenziell betreffendes Anliegen war die Forderung nach ,Pressfreiheit'“, Holger Jacob-Friesen, Profile der Aufklärung, Friedrich Nicolai - Isaak Iselin, Briefwechsel (1762-1782), Bern/Stuttgart/Wien, Haupt, 1997, S. 63.

17. Edict die Religions-Verfassung in den Preußischen Staaten betreffend. De Dato Potsdam, den 9. Julii 1788; Erneuertes Censur-Edict für die preußischen Staaten exclusive Schlesien. De dato Berlin, den 19. December 1788.

18. „German an Lichtfreund (böse Aspekten)“, HB II, 1788, S. 12-16.

19. Ibid., S. 12 . 
ungekünstelt an - ungeputzt, um mit Gellert zu reden - wie es in Briefen sein sollte. ${ }^{20}$ Der Aufsatz besteht aus einem Brief im Briefe; German warnt Lichtfreund: „Hören Sie, was mir dieser Tage ein Freund aus Berlin schreibt [...]“. ${ }^{21}$ Der Brief des Freundes aus Berlin wird im Brief von German an Lichtfreund in Anführungsstrichen zitiert. Man behauptet, steht darin, es wäre dem König ein Plan vorgelegt worden, eine Gewissensinquisition zu errichten. Somit wären Toleranz und Publizität zu Grabe - durch einen der sonderbarsten Züge des Schicksals ginge die Toleranz gerade da unter, wo sie entstand. ${ }^{22}$ (Eine deutliche Anspielung auf Friedrich II. ${ }^{23}$, der schon 1740 verkündet hatte, in seinem Staate könne jeder „nach seiner Fasson“ selig werden.) Allerdings wisse man noch nicht, ob Friedrich Wilhelm II. dieses traurige Projekt genehmigen werde. Das Geschäft sei ins „tiefste Geheimnis gehüllt“ und werde mit „großer Intrige behandelt“ ${ }^{24}$ (wiederum eine deutliche Anspielung, da Friedrich II. Wöllner einen „intriganten Pfaff“ ${ }^{25}$ nannte). Aber nach den Aspekten zu urteilen (der Titel des Briefes, „böse Aspekten“, bedeutet soviel wie Ahnung), machten sich die Urheber einige Hoffnung. Schon zeigten sich gewisse Funken einer unter der Asche liegenden Verfolgung. ${ }^{26}$ Somit enden die Zitate des Briefes aus Berlin. Nun wendet sich German erneut direkt an Lichtfreund: „Nun wissen wir also, worauf der Lärm vom Crypto-Katholizism zielte. Er sollte auf diesen Schlag vorbereiten. Er sollte der Verfolgung einen Anstrich geben. Diese Neuigkeit mag diejenigen heilen, die am Berliner Wurm leiden.“ ${ }^{27}$ Die Berlinische Monatsschrift hatte 1785 Berichte über die geheime Proselytenmacherei der Katholiken unter den Protestanten veröffentlicht. Den Rosenkreuzern und Freimaurern wurde vorgeworfen, dass sie Krypto-Katholizismus (insbesondere den Marienkult) betrieben. Die Geheimbünde erschienen folglich als ideales Forum für die geheime Unterwanderung und Aushöhlung des Protestantismus durch die katholische Lehre und jesuitische Umtriebe. ${ }^{28}$ „Und bei solchen Umständen mag man noch von Pfaffenzwang schwätzen, über Rom, Wien, Köln usw. schreien?“ fragt German rhetorisch. ${ }^{29}$ Hatte Wekhrlin sofort gegen das Religionsedikt

20. „Christian Fürchtegott Gellert hat seinen im 18. Jahrhundert maßgeblichen Traktat über den ,Guten Geschmack in Briefen' (1751) folgendermaßen eingeleitet: „Das erste, was uns bei einem Briefe einfällt, ist dieses, dass er die Stelle eines Gesprächs vertritt. Dieser Begriff ist der sicherste. Ein Brief ist kein ordentliches Gespräch [...] aber er vertritt doch die Stelle einer mündlichen Rede, und deswegen muss er sich der Art zu denken und zu reden, die in Gesprächen herrscht, mehr nähern als einer sorgfältigen und geputzten Schreibart.'“ Jacob-Friesen, Profile der Aufklärung, S. 17.

21. HB II, 1788, S. 12.

22. Ibid., S. 13.

23. Über „Das Problem der Toleranz bei Friedrich dem Grossen“ siehe Frank Lothar Kroll, Das geistige Preußen: zur Ideengeschichte eines Staates, Paderborn, Schöningh, 2001, S. 11-31.

24. $H B$ II, 1788, S. 13.

25. Christina Stange-Fayos, Lumières et obscurantisme en Prusse. Le débat autour des édits de religion et de censure (1788-1796), Bern, Lang, 2003, S. 72.

26. HB II, 1788, S. 13.

27. Ibid., S. 14. Hervorhebung. C. S.-F.

28. Kemper, Missbrauchte Aufklärung?, S. 87.

29. HB II, 1788, S. 14. 
Stellung bezogen, dauerte es in Sachen Krypto-Katholizismus viel länger, bis er sich mit den Berlinern einigen konnte. ${ }^{30}$ Er ärgerte sich über die Überheblichkeit des Nordens, der behauptete, das Licht der Aufklärung sei noch nicht bis in den Süden gedrungen. German schließt seinen Brief wie folgt: „Lassen Sie uns, Lichtfreund, immer an der Neuigkeit meines Korrespondenten zweifeln. Man sagt zwar, Wilhelm Friedrich der Zweite wäre gut; aber er ist nicht schwach." ${ }^{31}$ Allerdings irrte German-Wekhrlin sich, wenn er hoffte, die negative Reaktion der aufgeklärten Öffentlichkeit könne den König davon abhalten, das Edikt zu erlassen. Nicht auf die öffentliche Meinung hörte Friedrich II., sondern auf seine konspirativen Minister. 1788 wurde Johann Christoph von Wöllner Staatsund Justizminister sowie Chef des geistlichen Departements. Ab dem Moment übernahm er offiziell das „Generalkommando im Kampf gegen die Aufklärung“, wie er sich selber ausdrückte.

Als die Zeitschrift den nächsten Brief zu dem Thema veröffentlichte, „Erast an Argant (Gewissenssachen)“32, war das wöllnersche Edikt bereits erlassen worden. Erast (d.i. Wekhrlin) gibt vor, seinem Freund Argant ein Original zu senden, das völlig zum Seitenstück neben das „Berliner Glaubensedikt“ passe. Das Dokument, ein Zirkelbrief aus Madrid vom 4. März 1788, ist unterzeichnet „Graf Florida Bianca, Minister“ (einem der berühmtesten Aufklärern unter Karl III.). Er verbreitet einen königlichen Erlass, der notwendig erscheint, denn:

Gewisse geistliche Personen, ebenso ehrwürdig von der Seite ihres Amts als ihres Eifers und ihrer Einsichten, haben der Regierung sehr ernsthafte Vorstellungen wegen der offenbaren Gefahr gemacht, in welcher das Gewissen des Volks schwebt, in dem man solchem mittelst verschiedener, ihrer verdächtigen, dem Sinn des Evangels und anderer heiliger Schriftsteller widersprechenden Moral halber berüchtigten Schriften, nach besagte heillose, auf dem Umsturz des Reichs, der Kirche und der Sitten abzielende, und zu denen ungeheuer ersten Verbrechen veranlassende Sätze beizubringen suche. ${ }^{33}$

Der Trick des Briefes im Brief wird erneut benutzt; die mise en abyme fand man schon bei Plato. Wie das nach seinem Verfasser benannte preußische Religionsedikt besteht der spanische Erlass aus 14 Punkten. Erast bezeichnet ihn als „Bußpredigt, voll Salbung und Licht“ und gibt vor, sich darüber zu wundern, dass er keinen Beifall fand. Er behauptet ferner, der Zirkelbrief des spanischen Ministers bilde keine Satire des Berliner Religionsedikts: dazu sei die Ähnlichkeit zwischen beiden zu groß. ${ }^{34}$ Man bewundert die sokratische Ironie Wekhrlins, der ein regelrechtes Täuschungsmanöver vollzieht (wozu auch die Nennung Florida Biancas gehört, das Gegenstück zu dem, als der er vorgestellt wird $^{35}$ ). Dieser Brief sei eher ein Zeugnis dafür, dass der Gewissenszwang unter

30. Mondot, Wilhelm Ludwig Wekhrlin, S. 678.

31. HB II, 1788, S. 16.

32. „Erast an Argant (Gewissenssachen)“, HB III, 1788, S. 324-333.

33. Ibid., S. 325.

34. Ibid., S. 333.

35. Für diesen Hinweis bedanke ich mich bei Thomas Bremer. 
allen Himmel und allen Gestalten verhasst, und die Folgen davon überall analog seien, schließt Erast. ${ }^{36}$

Viele schriftliche Reaktionen folgten dem Erlass des Religionsediktes vom 9. Juli $1788 ;^{37}$ meist ernste Abhandlungen, philosophische Traktate über die Grenzen der Aufklärung aber auch einige Schmäh- und Spottschriften (insb. von K. F. Bahrdt, Karl Venturini, Titus H. Henke) ${ }^{38}$. Die Verstärkung der Zensur hatte nur Eines bewirkt: die Anzahl der Presseerzeugnisse explodierte förmlich... Eine Phase der kritischen Selbstprüfung wäre nach dem Tode Friederichs II. von Seiten der Aufklärung durchaus erwünscht gewesen. Allein die provokative Art mit der Wöllner und seine Rosenkreuzhelfer den Krieg gegen die Aufklärung initiierten, verhinderte eine konstruktivere Form der Auseinandersetzung. ${ }^{39}$ Auch der Ton der Hyberboreischen Briefe spitzte sich in Bezug auf die preußischen Maßnahmen zu. Die einschlägige Forschung vermutet, dass nun nicht mehr Wekhrlin selber, sondern Karl von Knoblauch zu Hatzbach (1756-1794), sein ständiger Mitarbeiter, die Aufsätze redigierte. ${ }^{40}$ Dieser war fast 30 Jahre jünger als Wekhrlin. ${ }^{41}$ Er war studierter Jurist und publizierte anonym viele Artikel in den bekannten Zeitschriften der Epoche, u.a. im Teutschen Merkur. Außer Wekhrlin und Wieland stand er auch mit Lichtenberg in Kontakt. Er war ein Kritiker von Kirche und Religion. ${ }^{42}$ Während seines Jurastudiums in Göttingen wurde er Freimaurer; dann gehörte er zur deutschen Union von Karl Friedrich Bahrdt. Knoblauchs Stil und Weltanschauung sind für die Spätaufklärung repräsentativ; er war insbesondere beeinflusst durch die Philosophie Holbachs, Diderots, Helvetius. ${ }^{43}$ Man kann ihn als Mitredakteur der Hyperboreischen Briefe betrachten.

1789 erschienen zwei weitere Briefe, d.h. Aufsätze, die in die Debatte um das Religionsedikt und seine Folgen eingreifen, diesmal also aus der Feder Knoblauchs. Zunächst gibt er vor, „Lindor [schreibe] an Araminthe“44, die er

36. Ibid., S. 333.

37. „Hier wurde nicht um eine begrenzte politische Maßnahme gestritten, hier stand vielmehr die preußische Aufklärungspolitik der letzten 40 Jahre grundsätzlich zur Disposition.“ (Kemper, Missbrauchte Aufklärung?, S. 60).

38. Stange-Fayos, Lumières et obscurantisme, S. 369-379.

39. Kemper, Missbrauchte Aufklärung?, S. 61.

40. Mondot, Wilhelm Ludwig Wekhrlin, S. 396-397.

41. Er gehörte der zu Hatzbach im früheren Kurhessen angesessenen ritterschaftlichen Familie des Namens an, siehe Karl Damian Achaz von Knoblauch zu Hatzbach, „Knoblauch, Karl von“, in Allgemeine Deutsche Biographie, Bd. 16 (1882), S. 307-308.

42. Er wurde von der früheren Forschung als Atheist und Materialist betrachtet, siehe Otto Finger, „Karl von Knoblauch - ein deutscher Atheist des 18. Jahrhunderts“, in Deutsche Zeitschrift für Philosophie 6, 1958, S. 924-984.

43. Jean Mondot, "Carl von Knoblauch ou les audaces religieuses et politiques d'un esprit fort ", in Roland Krebs (Hrsg.), Recherches nouvelles sur l'Aufklärung, Reims, Presses universitaires, 1987, S. 43-59.

44. „Lindor an Araminthe“, HB IV, 1789, S. 299-315. 
überschwänglich begrüßt: „Die Musen küssen sie, meine Freundin!“45 Er will ihr aus dem „Sapphischen Zirkel“ berichten (diese Fiktion verweist zugleich auf die Realität der Lesegesellschaften im 18. Jahrhundert) ${ }^{46}$, den ein Schöngeist namens „Abbé Palmerin“ eingerichtet hat (als niederer katholischer Weltgeistlicher wird der Abbé oft im Theater von Molière verspottet). Er empfängt die Abendgesellschaft in seiner eleganten Wohnung. „Alles was denkt und empfindet, d.h. die schöne Welt, hat sich einschreiben lassen. Es sind Vorlesungen über die schönen Wissenschaften, über Politik, Philosophie, Literatur, und was immer sie wollen." ${ }^{47}$ Die Abende verlaufen nach einem festen Schema: eine Dame (denn das Auditorium besteht aus beiden Geschlechtern) führt abwechslungsweise das Präsidium. Der Abbé liest vor, die anderen hören zu. Wenn die „Lektion“ geendigt ist, küsst man die Büste der Sappho (welcher das Institut „geheiligt ist“) und setzt sich an Spieltische... Mit den Namen aus der französischen bzw. italienischen Komödie knüpft der Verfasser an den vorigen Brief an Argant an. Lindor teilt Araminthe mit, der Abbé habe für die nächste Woche eine ernsthafte Materie zum Eingang versprochen. „Wollen Sie wissen wovon er handelte. Hier ist die Vorlesung auf ein Haar breit.“ " ${ }^{48}$ Ist die Struktur des ersten Satzes zwar die einer Fragestellung (s. Syntax), so sagt das abwesende Fragezeichen doch viel über seinen rhetorischen Charakter aus: dieser erste Satz ist ein Signal Lesegesellschaft und Vorlesung sind Fiktionen, die Vorlesung ist einem Brief beigefügt ist (ähnlich dem vermeintlichen Rundschreiben des spanischen Ministers, wieder eine mise en abyme). Der Publizist versteckt sich hinter mehreren fiktiven Personen, erscheint also selber nicht als Verfasser. Der Rest des Aufsatzes - vom Brief säuberlich durch zwei Striche getrennt und auf einer neuen Seite beginnend - besteht aus der Anrede des Gastgebers („Meine Dames und Herren!“ usw.) Der Leser soll den Eindruck gewinnen, dass diese Lesegesellschaft tatsächlich existieren könnte - und wird in den Kreis mit einbezogen. Nach einer kurzen, amüsanten Einleitung geht der Abbé in medias res:

In Pruthenien gibt es, wie sie gehört haben werden, ein Gesetz, welches die Priester ein für alle Mal an die Leerformeln fesselt, welche vor Jahrhunderten von anderen Priestern, die, wie diese, auch an die Wand pissten, und irren konnten, nach Maßgebung der damaligen Kenntnisse, Begriffe, und Einsichten festgesetzt worden waren. Wer in seinen Vorträgen von diesen Formeln abweicht, oder mitunter gewisse Meinungen äußert, welche das Gesetz für längst widerlegte Irrtümer erklärt, dem droht das Gesetz mit der Kassation. ${ }^{49}$

45. Ibid., S. 299. Über den Freundschaftsbegriff der Aufklärung, Anrede und Schlussformel in Briefen, Freundschaftsepitheta als Indikatoren für Vertiefung des Dialogs und Verbundenheit siehe Jacob-Friesen, Profile der Aufklärung, S. 19-20. Das von den Stürmern und Drängern praktizierte Duzen lehnten die Aufklärer ab.

46. Otto Dann (Hrsg.), Lesegesellschaften und bürgerliche Emanzipation, München, Beck, 1981.

47. HB IV, 1789, S. 300. Hervorhebung. C. S.-F.: „Diese Vereine [...] dienen einzig dem Bedürfnis der bürgerlichen Privatleute, als räsonierendes Publikum Öffentlichkeit zu bilden.“ Jürgen Ziechmann, Panorama der Fridericianischen Zeit, Bremen, Ziechmann, 1985, S. 272.

48. HB IV, 1789, S. 300.

49. Ibid., S. 302. 
Pruthenien steht kaum verhüllt für Preußen und man erkennt mühelos den siebten und zentralen Paragraphen des Religionsedikts. ${ }^{50}$ Triviale Elemente im Diskurs („Priester [...] die [...] an die Wand pissten“) stehen in Opposition zum (fiktionalen) Kontext: literarischer Zirkel, erlesenes Publikum (Damen!) und Behandlung eines ernstes Themas. Dies erinnert an den Stil der Popularphilosophen. Mit ihrer ungehemmten Neigung, Tradiertes zu kritisieren und zu verwerfen ohne etwas Neues an dessen Stelle zu setzen, übten die Popularaufklärer eine verunsichernde Wirkung aus. ${ }^{51}$ Natürlich respektiere man die Gesetzgeber, versichert der Abbé daher, doch seien diese auch nur Menschen. Die Kritik, die er als „freimütige Untersuchung und Beurteilung menschlicher Dinge“ definiert, sei Sache der Philosophen. ${ }^{52}$ Ferner zitiert Palmerin-Knoblauch Kant frei nach der Kritik der reinen Vernunft: nichts könne der Prüfung der Kritik entzogen werden, weder die Religion durch ihre Heiligkeit noch die Gesetzgebung durch ihre Majestät. ${ }^{53}$ Das kultivierte Publikum erkannte die Huldigung auch ohne genauere Angabe. Sich über das Edikt selbst, sowohl über die Religion zu äußern, gehöre zu den angeborenen, unveräußerlichen Menschenrechten: "Wir haben Gottlob! Keine Bastille, keinen Scheiterhaufen, und kein Schiffziehen zu fürchten. Unsere Regierung ist zu weise und - zu gut." ${ }^{54}$ Hier besteht die Ironie aus hinter Ernst verstecktem Spott, mit dem man das Gegenteil von dem ausdrückt, was man meint, seine wirkliche Meinung aber durchblicken lässt... Ein gefährliches Spiel, da hier über die Machthaber gespottet wird. ${ }^{55}$ Ein Priester, der zu ehrlich wäre, gegen seine eigene Überzeugung zu lügen und zu viel Ehrgeiz besäße, seine Vorträge dem Spott der Denker aussetzen zu wollen, müsste notwendig seine Predigten mit dem „Grade der Kenntnis und Aufklärung“ und mit dem Tone der Philosophie seiner Zeit übereinstimmig machen, behauptet Palmerin. (Aufklärung wäre folglich etwas Messbares). „Er dürfte nicht länger Dinge für ausgemachte Wahrheit geben, welche den größten Denkern unglaublich, oder doch zweifelhaft und unerweislich scheinen. " ${ }^{56}$ Dies ist eine Kritik an der geoffenbarten Religion, die das Religionsedikt (siehe \$7) wieder durchsetzen wollte. Als Theologen müssen Kirchenmänner den wissenschaftlichen und philosophischen Fortschritt

50. „Merkwürdiges Edict Sr. Königl. Majestät von Preussen, die Religionsverfassung in den preussischen Staaten betreffend“, in Acten, Urkunden und Nachrichten zur neuesten Kirchengeschichte, Bd. 1, 6. St., Weimar, Schneider, 1788, § 7, S. 469-471. Von „Cassation“ ist in $\S 8$ ausdrücklich die Rede, siehe Stange-Fayos, Lumières et obscurantisme, S. 402.

51. Kemper, Missbrauchte Aufklärung?, S. 65. Siehe auch die zeitgenössische Broschüre Wie ist es möglich geworden, Aufklärung und Aufklärer verhasst zu machen?, Von einem Wahrheitsfreunde, Auf der Insel Felsenburg [= Hamburg], 1789.

52. HB IV, 1789, S. 303. Der Ursprung der Kritik liege in der sokratischen Schule, ibid. S. 309-310.

53. $H B$ IV, 1789, S. 303.

54. Ibid.

55. "Il faut tenir compte du contexte rhétorique dans lequel ces idées furent défendues. Dans la société d’Ancien régime, le discours approbateur allait pour ainsi dire de soi. La critique était beaucoup moins courante que dans nos sociétés libérales modernes. Elle se détachait par conséquent bien davantage sur la fadeur convenue des lieux communs bien-pensants. " (Mondot, Wilhelm Ludwig Wekhrlin, S. 679).

56. HB IV, 1789, S. 307. 
beachten; im Zeitalter der Aufklärung könne es keine Orthodoxie mehr geben. ${ }^{57}$ Dass der Gesetzgeber den Glauben nicht verordnen kann, ist eine Anspielung auf die Broschüre des Juristen Hufeland, die großen Erfolg in der Debatte um das preußische Religionsedikt gehabt hatte. ${ }^{58}$ Ferner führt der Abbé Montesquieu an („den großen Verfasser vom Geist der Gesetze“), indem er behauptet, man könne von dem politischen Nutzen einer Religion nicht auf ihre Wahrheit schließen. Dass die Religion unentbehrlich für die bürgerliche Gesellschaft ist, sei keine auf geprüften Argumenten beruhende Tatsache. ${ }^{59}$ Palmerin stützt sich auf eine historische Argumentation, um zu zeigen dass die Religion ein politisches Instrument ist, um die Ordnung aufrecht zu erhalten. „Die ersten Römer waren keine Christen, aber, wie man nicht leugnen kann, gerechter, mäßiger, und von einfacheren, reineren Sitten, als wir“.60 Die schlimmsten Verbrechen, Akte der Unzucht, etc., fanden im Mittelalter (wo die Vernunft unter dem Gehorsam eines blinden Glaubens gefangen genommenen war) weit häufiger statt, als in den Zeitaltern der Philosophie und des Skeptizismus, „das beweist die Geschichte“. ${ }^{61}$ Dieser Glauben in die Geschichte als Wissenschaft war im Laufe des 18. Jahrhunderts in Deutschland stärker geworden und ist für die Aufklärung charakteristisch. ${ }^{62}$ Ein echter Philosoph sei ein Mann, der „die menschlichen Dinge, ohne Brille, ohne Vorurteile, mit der Fackel der Geschichte und der Erfahrung in der Hand, untersucht."63 Dieser Diskurs konnte ein noch sehr gläubiges Publikum provozieren und wirtschaftliche Konsequenzen für die Zeitschrift mit sich bringen. Noch zehn Jahre später verlor Fichte die Gunst des Publikums und fand sie nie wirklich wieder, da er des Atheismus beschuldigt und aus Jena verjagt wurde. Die Redakteure der Hyperboreischen Briefe wanderten auf einem äußerst schmalen Grat, indem sie Philosophie gegen Theologie, Aufklärung gegen Religion stellten und behaupteten, die Moral liege in der Vernunft und die aufgeklärte Gesellschaft brauche keine Religion, diese nur zu Machtzwecken diene. Auch bildet der nächste Brief eine scharfe Kritik des geistlichen Standes.

Dieser letzte Beitrag der Hyperboreischen Briefe zur Diskussion um das preußische Religionsedikt ist zugleich der gewagteste, enthält er doch ein Plädoyer für den antichristlichen Rationalisten Carl Friedrich Bahrdt. Er trägt den Titel „Klindor an Aramintha“ (Untertitel „zwote Lektion siehe viertes Bändchen Nummer 34“") ${ }^{64}$ : Abbé Palmerin beauftragt angeblich Klindor, Aramintha seine

57. Ibid.

58. Gottlieb Hufeland, Ueber das Recht protestantischer Fürsten unabänderliche Lehrvorschriften festzusetzen, Iena, Cuno, 1788.

59. HB IV, 1789, S. 308.

60. Ibid., S. 310.

61. Ibid., S. 311.

62. Horst Möller, Aufklärung in Preußen. Der Verleger, Publizist und Geschichtsschreiber Friedrich Nicolai, Berlin, Colloquium, 1974, Kapitel 6, „Aufklärung und Geschichte“, S. 322 ff.

63. $H B$ IV, 1789, S. 314.

64. „Klindor an Aramintha. Zwote Lektion“, HB V, 1789, S. 305-316. 
neue Lektion zu übersenden. Die Fiktion des „Sapphischen Zirkels“ wird also weiter gesponnen. Nach dieser kurzen Einleitung folgt sofort die zweite Vorlesung. Sie wird typographisch weniger deutlich abgetrennt als im vorigen Brief (nur durch einen halben Strich) - sinkt etwa die Vorsicht der Redakteure? Seit der Reformation habe die Menschheit sich von der Religion emanzipiert und das Ansehen der Geistlichen sei gesunken, erklärt der Abbé. Da die Obrigkeiten ihnen nicht mehr ihren Arm leihen wollten, konnten sie nichts mehr tun, als beklagen, dass der „Unglauben“ - d.h. Philosophie und Toleranz - bis zu den Thronen der Könige durchgedrungen sei. ${ }^{65}$ Friedrich der Zweite wird bezeichnenderweise als „König des Jahrhunderts“ bezeichnet ${ }^{66}$, als „Friedrich der Unerreichte“ ${ }^{67}$, als „Friedrich der Weise“ ${ }^{68}$, als „der gekrönte Philosoph, der unsterbliche Friedrich“ ${ }^{69}$. Die Steigerung zeigt Knoblauch, wie Wekhrlin, als Bewunderer Friedrichs und dessen Toleranz - bzw. Antiklerikalismus (wie Lessing es schon 1769 in einem Brief an Friedrich Nicolai entlarvte). Religionsedikte werden von Abbé Palmerin als Regression betrachtet: sie vermehren bloß die Zahl der Heuchler und legen dem Untersuchungsgeist drückende Fesseln an. Dabei gehen religiöse Überzeugungen der Individuen den Staat seiner Meinung nach nichts an. ${ }^{70}$ Daraufhin wir die Fiktion eines neuen Briefes eingeflochten, indem Palmerin behauptet, einen Brief erhalten zu haben: Sein Freund Philint aus Wien habe ihm neulich geschrieben, um sich über die Verstärkung der Zensur zu beklagen. ${ }^{71}$ Verordnungen wie das Religionsedikt seien Messer in den Händen der Regierungen (das Messer dient, ähnlich der Schere, als Metapher für die Zensur). Philint macht seiner Empörung über diese gesetzliche Maßnahme im Brief an Freund Palmerin jedoch Luft, ohne ein Blatt vor den Mund zu nehmen: „Polizeiaufseher, Gedankenspione, Gewissensjäger - traurige Springfeder des Despotismus! Betrübter Beweis des Misstrauens der Obrigkeit gegen das Publikum! Sicheres Mittel, aus offenen Bürgern Duckmäuser und Heuchler zu machen!“72 Dieser Brief aus Wien mutet zunächst befremdlich an, da die Zensur (durch Joseph II.) dort doch kürzlich gelockert worden war. ${ }^{73}$ Dieser Widerspruch löst sich auf, bedenkt man, dass in Wien kürzlich Bahrdts kontrovers aufgenommenes Lustspiel über das Religionsedikt - anonym - erschienen war. Es hatte großen Erfolg und wurde fünfmal neu aufgelegt; Bahrdt wurde von seinem eigenen Sekretär angezeigt und von der Polizei verhaftet, obwohl er die Autorschaft leugnete. ${ }^{74}$ Unvermittelt stimmt der Abbé einen Lobgesang auf Friedrich II. an: „Friedrich der Unerreichte

65. Ibid., S. 308.

66. Ibid., S. 309.

67. Ibid., S. 311.

68. Ibid., S. 312.

69. Ibid., S. 314.

70. Ibid., S. 309-310.

71. Ibid. Der Name stammt aus dem Menschenfeind (Misanthrope) von Molière.

72. Ibid., S. 311.

73. Lesli Bodi, Tauwetter in Wien. Zur Prosa der österreichischen Aufklärung, 1781-1795, Frankfurt a.M., Fischer, 1977.

74. Palmerin erinnert an die Verhaftung und den Prozess Bahrdts, HB V, 1789, S. 313-315 (eine abenteuerliche Geschichte, die das Publikum faszinieren musste). 
ließ jeden reden und schreiben. Er fürchtete die Satire nicht, weil er überzeugt war, sie nicht zu verdienen." ${ }^{75}$ Er vertraute dem aufgeklärten Publikum, welches in der Lage war, über den „Lästerer“ zu urteilen. ${ }^{76}$ Laut Palmerin gab es keine Zensur, keine polizeiliche Verfolgung im friderizianischen Preußen, was zwar nicht der Wahrheit entsprach ${ }^{77}$, dem (verantwortungsbewussten) Publikum, welches er in Szene setzt, jedoch schmeicheln musste. All dies, so unvermittelt es zunächst auch erscheint, dient der rhetorischen Vorbereitung Bahrdts Verteidigung. Tatsächlich fügt der Abbé hinzu: „Inzwischen sehen wir diese Regel durch eine ganz neue Begebenheit unterbrochen, worüber das Jahrhundert Friederichs des Weisen seufzt. Man schreibt über das berühmte Religionsedikt eine Fratze. “" ${ }^{78}$ In der Tat mokierte sich Bahrdt in einer Weise über das Edikt und dessen Urheber ${ }^{79}$, die ihm den Tadel der aufgeklärten Publizisten einbrachte ${ }^{80}$, da er ja gerade das Schreckbild des gottlosen Religionsspötters verkörperte, für die Wöllner die Aufklärer ausgeben wollte. ${ }^{81}$ Der Abbé selber scheint das Stück zunächst zu verurteilen: der Philosoph, doziert er, habe das Recht auf ernste und bescheidene Prüfung und würde nie die Ehrfurcht gegen König und Minister außer Augen lassen. ${ }^{82}$ Beugen sich die Hyperboreischen Briefe mit dieser Aussage dem Konsens über die Presse als mächtige Waffe, die vorsichtig zu handhaben sei? ${ }^{83}$ Nein: es folgt vielmehr ein Plädoyer für die uneingeschränkte Pressefreiheit. Die Zeichensetzung besteht in diesem Absatz ausschließlich aus Frage- und Ausrufezeichen; der dynamische Stil stellt einen Dialog mit dem Leser her: „Sie haben recht: jeder unter uns hat inalienable Ansprüche auf bürgerliche Sicherheit und Freiheit, solange er die allgemeine Sicherheit nicht stört. Aber tut man dies, wenn man eine elende Komödie schreibt? Befördert man

75. $H B \mathrm{~V}, 1789$, S. 311.

76. Ibid., S. 312.

77. Martin Welke, „Das Pressewesen“, in Ziechmann (Hrsg.), Panorama, S. 425-436.

78. $H B$ V, 1789, S. 312-313.

79. Eine kurze Leseprobe dürfte ausreichend sein: „Gott! Ein besoffenes Schwein! Der Konzipient eines Religionsedikts!“ Damit ist J. C. von Wöllner genannt. Das Religions-Edikt. Ein Lustspiel in fünf Aufzügen. Eine Skizze. Von Nicolai dem Jüngern, Gedruckt durch Johann Michael Bengel, Thenakel [Gera] [Wien, Wucherer], 1789, Akt I, Szene 5.

80. Bahrdt sei „[...] einer der verworfensten Menschen, ein warnendes Exempel der unglücklichsten, durch Eigendünkel, Leichtsinn und Wohllust bewirkten Verwilderung der Sitten, und Verwahrlosung guter Talente.“ Allgemeine Deutsche Bibliothek, 114/2, Kiel, 1793, S. 85-86.

81. „Das die Politik Wöllners dominierende Schreckbild des gottlosen Religionsspötters und gefährlichen Aufklärers zielte in erster Linie auf seine unmittelbaren Gegner in Berlin, bei denen es sich ja keineswegs um radikale Positionen einnehmende, sondern um gemäßigte und durchweg staatstragende Vertreter der Aufklärungsbewegung handelte ; dies gilt für die Mitglieder des Oberkonsistoriums und den Minister von Zedlitz ebenso wie etwa für Nicolai, Biester oder Gedike. In Vergleich zu diesen gleichsam institutionalisierten Aufklärern erscheint Karl Friedrich Bahrdt als der eigentliche Dissident, als der er, nicht zuletzt im eigenen Lager, auch wahrgenommen wurde.“ Christoph Weiss, „Krieg gegen die Aufklärer“, in Gerhard Sauder/ Christoph Weiss (Hrsg.), Carl Friedrich Bahrdt, 1740-1792, St. Ingbert, Röhrig, 1992, S. 329.

82. $H B \mathrm{~V}, 1789$, S. 313.

83. Siehe E. F. Klein, „Ueber Denk- und Druckfreiheit. An Fürsten, Minister und Schriftsteller“, in Berlinische Monatsschrift, 1, 1784, S. 312-329. 
dadurch den Straßenraub, die Empörung, die Mordbrennerei? Nein. " ${ }^{84}$ Die Presse ist nicht umstürzlerisch, was die Forderung nach Pressefreiheit rechtfertigt. Das Gesetz seinerseits stellt Palmerin-Knoblauch als „Produkt der Autorschaft und der Literatur der Regierung“ dar, was ihm erlaubt, es als „Gegenstand der Kunstrichter“ zu erklären. ${ }^{85}$ Somit ist es der Zensur des Publikums unterworfen - anders ausgedrückt: die Zustimmung des Publikums legitimiert das Gesetz. Der Begriff „Öffentlichkeit“ war am Ende des 18. Jahrhunderts politisch aufgeladen, Öffentlichkeit als Forum diskursiver Wahrheitsfindung stand als richtende Instanz oberhalb der staatlichen Legislative. ${ }^{86}$ Doch diese Öffentlichkeit war noch im Entstehen begriffen; man hat es hier mit einer Utopie der Aufklärung zu tun (die Gesetzgebung steht ideal über den Machthabern). Die Gewaltentrennung stand in Wirklichkeit nicht auf der Tagesordnung, im Gegenteil (siehe Maßnahmen wie das wöllnersche Religionsedikt...) Trotzdem suggeriert Knoblauch-Palmerin, dass das Edikt schon zu stark kritisiert wurde, um in Kraft zu bleiben: die Presse spielt für ihn eine quasi institutionelle Rolle, als „Gegengewalt“ (Montesquieu) die der absolutistischen Macht Schranken setzt. ${ }^{87}$ Die strenge Behandlung Bahrdts - dieser wurde zu zwei Jahren Festungshaft verurteilt - zeigt, dass die Obrigkeit sich davon nicht einschüchtern lies, was die Hyperboreischen Briefe als Ungerechtigkeit anprangerten. Wenige zeitgenössische Publizisten gingen so weit: man findet in der Debatte um das Religionsedikt eine einzige bezeichnenderweise anonyme Verteidigung des Volksaufklärers Bahrdt. ${ }^{88}$ Die Hyperboreischen Briefe vertraten somit gegen die meisten anderen aufgeklärten Presseorgane eine radikale Meinung: ${ }^{89}$

Nochmal, meine Dames und Herren: der Doktor verfehlte sich. Er beleidigte Klugheit und Anstand. Er prostituierte sich und die Musen. Allein Dies hätte Strafe genug für ihn sein sollen. Die Justiz, wollte sie Philosophie zeigen, hätte sich dabei begnügen sollen. Sie hätte eine sublime Verachtung affektieren, und, wie unser Freund Damis ${ }^{90}$ spricht: dem Verfasser das, was er sagte, verzeihen sollen für das, was er nicht sagte. ${ }^{91}$

84. $H B \mathrm{~V}, 1789$, S. 315.

85. Ibid.

86. Dirk Kemper, „Obskurantismus als Mittel der Politik. Johann Christoph von Wöllners Politik der Gegenaufklärung am Vorabend der französischen Revolution“ in Christoph Weiss/Wolfgang Albrecht (Hrsg.), Von „Obscuranten“ und „Eudämonisten“. Gegenaufklärerische, konservative und antirevolutionäre Publizisten im späten 18. Jahrhundert, St. Ingbert, Röhrig, 1997, S. 212.

87. Mondot, Wilhelm Ludwig Wekhrlin, S. 20.

88. [Degenhardt Pott], Anreden an die Richter des D. Bahrdt, von einem deutschen Manne H.v.L, o. 0, 1789.

89. Man bemerke die nachhaltige Feindseligkeit Knoblauchs insb. Friedrich Nicolai gegenüber: in seinem Aufsatz über Wekhrlin in der Allgemeinen Deutschen Biographie behauptet Knoblauch, Wöllner habe Nicolai aufgrund seiner früheren Mitarbeiterschaft bei der Allgemeinen Deutschen Bibliothek protegiert; dabei wurde Nicolais $A D B 1794$ aufgrund der Wöllnerschen Edikte verboten. (Es stimmt allerdings, dass Wöllner zwischen 1765 und 1780 aktiv an der $A D B$ mitarbeitete und gute Beziehungen zu Nicolai pflegte).

90. Damis war angeblich ein Reisegefährte des neupythagoreischen Philosophen Apollonius von Tyana.

91. $H B, 1789$, V, S. 316. 
Diese doppeldeutige Aussage erfordert eine Interpretation, ja gar klare Stellungnahme: der letzte Brief stellt ein doppeltes Plädoyer dar, für die absolute Pressefreiheit und für Bahrdt als Opfer der Obrigkeit.

Zusammenfassend kann daran erinnert werden, dass der Brief im 18. Jahrhundert ein Modephänomen war. Wekhrlin, der von seinen Publikationen leben musste, wählte diese Form wohl z.T. zunächst auch deshalb. Darüber hinaus befürwortete er einen freien Journalismus, der sich an das breite Publikum statt nur an Akademiker richtete: „Ich wähle Briefe - weil sich das Ding leicht weg lesen lassen soll“" schrieb er in der ersten Ausgabe der Hyperboreischen Briefe. ${ }^{92}$ In dieser Hinsicht kann man ihn als Volksaufklärer betrachten. ${ }^{93}$ Auch besa $\beta$ der Brief einen dialogischen Charakter: Die Aufklärer wollten insgesamt durch authentische literarische Form echte Kommunikation mit dem Publikum herstellen. Die Leser sollten am Reflexionsprozess der Publizisten teilnehmen ${ }^{94}$ und sich ihre eigene Meinung bilden. ${ }^{95}$ Die Art und Weise, in welcher die Hyperboreischen Briefe in der Tradition der epistolae obscurorum virorum (fingierte Briefe mit satirischer Absicht) das preußische Religionsedikt persiflierten und die Schnelle der Reaktion zeigen einmal mehr, dass der Süden dem Norden nicht nachstand, was den „Grad(e) der Aufklärung““96 betraf.

Die Struktur der Aufsätze (Briefe im Brief usw.) verweist auf die Briefkultur der Epoche: Briefe waren in der Tat nicht nur an einen Leser gerichtet, sondern wurden weitergeschickt, herumgereicht, vorgelesen. Man kann diesbezüglich von „Halböffentlichkeit“ des Briefverkehrs sprechen. ${ }^{97}$ Die Hyperboreischen Briefe wollten trotz Fiktion den Anschein der Realität wahren. Der Herausgeber stellte sich ausdrücklich als „Sammler“ der Briefe dar; somit war er juristisch abgesichert, denn er konnte für den Inhalt der Briefe (d.h. eigentlich: der veröffentlichten Aufsätze) nicht haften. Einerseits sollte das Briefliche der Fiktion Glaubwürdigkeit verleihen (Vorbild waren Pierre Bayles Pensées diverses sur la Comète, 1682), denn der Briefschreiber agiert spontan: " [il a] cet air libre qu'on se donne quand on écrit à un ami, mais non quand on destine un texte à

92. $H B, 1788$, I, S. 10.

93. "Ses efforts conscients pour être lu d'un vaste public n'ont donc pas été infructueux, du moins pendant un certain temps. Les critiques adressées à ses trop nombreux lecteurs témoignent qu'il avait réussi à toucher un public plus large que celui des revues traditionnelles. Wekhrlin a donc bien représenté un phénomène nouveau dans l'histoire du journalisme allemand." (Mondot, Wilhelm Ludwig Wekhrlin, S. 290. Hervorhebung C. S.-F.).

94. Hans-Erich Bödeker, „Aufklärung als Kommmunikationsprozeß“, in Aufklärung 2, Heft 2, 1987, S. 89-111.

95. Helmut Kiesel/Paul Münch, Gesellschaft und Literatur im 18. Jahrhundert. Voraussetzung und Entstehung des literarischen Markts in Deutschland, München, Beck, 1977, S. 166.

96. HB IV, 1789, S. 307.

97. Reinhard Nickisch, Brief, Stuttgart, Metzler, 1991, S. 51; Sibylle Schönborn, Das Buch der Seele: Tagebuchliteratur zwischen Aufklärung und Kunstperiode, Tübingen, Niemeyer, 1999, S. 14; Rafael Arto-Haumacher, Gellerts Briefpraxis und Brieflehre. Der Anfang einer neuen Briefkultur, Wiesbaden, Deutscher Universitätsverlag, 1995, S. 181. 
publication. ${ }^{98}$ Andererseits sollte „das Briefliche“, also die Fiktion des privaten bzw. (nur) halböffentlichen Diskurses, der öffentlichen Kritik ihre Schärfe nehmen. Letztlich war indirekte Kritik ein Mittel, die Zensur zu umgehen. ${ }^{99}$ Trotzdem wurde die Zeitschrift 1790 in fine verboten: sie erschien als zu demokratisch, fast schon ,jakobinisch“ (Jean Mondot). Vorerst jedoch milderte die „civilité épistolaire“ die ungewöhnlich scharfe politische Kritik rein formal ab und erhöhte somit ihre weitergehende Akzeptanz für das Aufklärungspublikum.

98. Hubert Bost, Pierre Bayle et la religion, Paris, PUF, 1994, S. 26.

99. Über die Verfahren der indirekten Kritik damals siehe Horst Möller, „Wie aufgeklärt war Preußen?“, in Hans-Jürgen Puhle / Hans-Ulrich Wehler, Preußen im Rückblick, Göttingen, Vandenhoeck \& Ruprecht, 1980, S. 193. 